\title{
Diagnosis and Surgical Management of Traumatic Cerebrospinal Fluid Oculorrhea: Case Report and Systematic Review of the Literature
}

\author{
Matthew Pease ${ }^{1} \quad$ Yvette Marquez $^{1} \quad$ Alex Tuchman $^{1} \quad$ Alex Markarian$^{2} \quad$ Gabriel Zada ${ }^{1}$ \\ ${ }^{1}$ Department of Neurosurgery, Keck School of Medicine of USC, Los \\ Angeles, California, USA \\ 2 Department of Otolaryngology, Keck School of Medicine of USC, Los \\ Angeles, California, USA \\ Address for correspondence Gabriel Zada, MD, Department of \\ Neurosurgery, USC, 1200 North State Street, Suite 3300, Los Angeles, \\ CA 90089, USA (e-mail: gzada@usc.edu). \\ J Neurol Surg Rep 2013;74:57-66.
}

\author{
Abstract \\ Keywords \\ - oculorrhea \\ - cranio-orbital fistula \\ - cerebrospinal fluid \\ - rhinorrhea \\ - traumatic brain injury \\ - surgical management \\ - pseudoepiphora \\ - cerebrospinal fluid \\ fistula
}

Background Oculorrhea, or cerebrospinal fluid leakage developing from a cranioorbital fistula, is a rare development following traumatic injury.

Case Report A 22-year-old man involved in a motor vehicle accident developed a blowout fracture of the left orbital roof penetrating the frontal lobe, inducing oculorrhea. He underwent a supraorbital craniotomy for removal of the bony fragment and skull base reconstruction using a pericranial flap.

Methods A systematic review of the database was performed to identify all prior cases of traumatic oculorrhea.

Results Twenty-two reported cases met inclusion criteria for subsequent analysis. Oculorrhea developed due to blunt and penetrating head injury in 14 (64\%) and 8 patients (36\%), respectively. The most common mechanisms were car accidents, stab wounds, falls, and gunshot wounds. Ocular signs and symptoms-including visual loss, ophthalmoplegia, and pupillary dysfunction-were commonly associated findings. Initial conservative management was successful in four patients. Thirteen patients underwent initial surgical intervention, and three additional patients required operative intervention following failed conservative treatment.

Conclusion Although oculorrhea rarely develops following severe orbital trauma, suspicion should nevertheless be maintained to facilitate more prompt diagnosis and management. The decision for conservative versus surgical management often depends on the severity of the fracture and dural injury.

\section{Introduction}

The Roman physician Galen first surveyed cerebrospinal fluid (CSF) leakage mechanisms when he described how fluid stored in the ventricles escaped through the nose. ${ }^{1}$ Modern medicine has since refined and broadened this understanding of CSF fistulas to recognize them as a potential complication of craniocerebral injury. The most common sites of traumatic CSF fistulae are the anterior and middle skull base, resulting in rhinorrhea or otorrhea. ${ }^{2}$ In addition, CSF can occasionally migrate into the orbit or through the scalp and into the eyelid and form a cranio-orbital fistula and pseudomeningocele, in which there is no outward leakage of CSF. In rare cases, however, CSF can transverse the orbit and exit via the eye, received

December 26, 2012

accepted after revision

March 18, 2013

published online

May 23, 2013 (c) 2013 Georg Thieme Verlag KG Stuttgart · New York
DOI http://dx.doi.org/ 10.1055/s-0033-1347902. ISSN 2193-6358. 
mimicking tear formation. Salame et al described this phenomenon as oculorrhea.*

A variety of mechanisms can cause orbital CSF fistulas, including orbital tumors with intracranial extension into subarachnoid space, orbital surgery that compromises the integrity of the cribriform plate, or craniocerebral trauma. The overall incidence of CSF fistulas following craniocerebral injury is 0.5 to $3 \%$, with rates upwards of $25 \%$ for midfacial injuries. ${ }^{4}$ Traumatic cranio-orbital CSF fistulas, however, are exceedingly rare, with fewer than 30 cases reported in the English literature. ${ }^{3}$

The paucity of reported oculorrhea cases, especially compared with the frequency of other types of CSF fistulas developing after traumatic injury, suggests that cranio-orbital fistulas are rare and may be underdiagnosed complications. Periorbital swelling from the accompanying head injury, misidentification of leaked CSF, and low clinical suspicion can delay proper identification of this entity, thereby potentially increasing the chance for hazardous complications including meningitis, intracranial hypotension, seizures, and encephalocele development. In the current study, we present the case of a young man involved in a motor vehicle accident who developed refractory oculorrhea in association with an orbital roof fracture that ultimately required surgical intervention. To further characterize the presenting features, diagnostic challenges, and treatment options for traumatic oculorrhea, we aimed to perform a systematic review of the literature pertaining to cranio-orbital fistulas.

\section{Materials and Methods}

A systematic review of the literature was performed in the database during the month of November 2012 to identify any articles published in the English language pertaining to traumatic oculorrhea. A search including the terms oculorrhea cranio-orbital CSF fistula, orbital fistula, and pseudoepiphora yielded 480 search results. Pertinent abstracts were screened. Trials were included in this review if the aim of the paper: (1) described clinical studies pertaining to mechanisms, diagnosis, and/or management of traumatic cranio-orbital fistulas and/or oculorrhea; (2) reported cases of traumatic cranio-orbital fistulas and/or oculorrhea; and (3) did not describe oculorrhea resulting from a nontraumatic orbital or lacrimal surgery. In addition, references of all included articles were reviewed to search for additional studies. Overall, 46 relevant studies meeting the criteria for preliminary analysis were identified. Of these, 24 were excluded for the following reasons: relevance, lack of clinical data reported, inability to acquire the original article, and lack of evidence of oculorrhea occurring. Following exclusion, 22 cases of traumatic oculorrhea were included in the review (-Table 1). Each study was thoroughly reviewed, and pertinent clinical data including

\footnotetext{
*For the purposes of this paper, oculorrhea is the symptom of outward leakage of CSF. A cranio-orbital fistula is any opening that allows communication between the cranial vault and the orbit.
}

demographics, mechanism and patterns of injury, diagnosis, treatment, and follow-up were extracted and entered in a database. Given the rarity and heterogeneity of cases, no meta-analyses were performed.

\section{Case Report}

A 22-year-old restrained male driver fell asleep while driving and struck a parked car, hitting his head on the steering wheel of his car. He did not lose consciousness. Although he had a previous history of seizures, he had been seizure free for 7 years without antiepileptic medications. The patient was transferred to the Keck-USC Medical Center for further care.

\section{Examination}

The blood pressure was $127 / 68$, pulse 64 , and respiratory rate was 16 . He was alert, oriented, and cooperative. A left forehead abrasion was evident, in addition to a 3-cm laceration on his left eyelid. The left eye was severely swollen and ecchymotic. Upon assisted opening of the left eye, the pupils were round, equal and reactive to light with a visual acuity of 20/20 in the right eye and 20/40 in the left eye. The following extraocular movements were noted in the left eye: 100\% adduction, $90 \%$ abduction, $80 \%$ infraduction, and 30\% supraduction. The right eye examination was normal. In addition, spontaneous clear fluid was noted to be draining from the eye, and was exacerbated when leaning the patient forward. No rhinorrhea or otorrhea was present on exam. The remainder of the cranial nerve and neurological examination was within normal limits.

A noncontrast, thin-slice computed tomography (CT) of the brain and orbits with coronal and sagittal reconstructions showed a displaced fracture involving the left orbital roof and lateral ethmoid sinus, with an 8-mm superior migration of the dominant fragment into the left gyrus rectus, and small amount of intracranial pneumocephalus, hyperdense hemorrhage, and edema (-Fig. 1). The left ocular globe appeared to be intact with a small amount of stranding within the intraconal space. A blowout fracture involving the orbital floor with prolapse of orbital fat and inferior rectus muscle into the maxillary sinus was also evident. A small amount of blood products were present within the maxillary sinus. The left lamina papyracea was fractured with hemorrhage into multiple ethmoid air cells. A moderate amount of soft tissue edema was noted overlying the left preorbital soft tissue.

Despite initial efforts at conservative management in an attempt to allow spontaneous resolution of the oculorrhea, fluid leakage from the eye persisted. Given the refractory oculorrhea, bony fragment extending into the frontal lobe, pneumocephalus, and risk for infection and/or seizure, surgical resection of the bony fragment and repair of the cranioorbital fistula was recommended.

\section{Operation}

The patient underwent a left frontal supraorbital craniotomy on hospital day 4. The patient was positioned in supine position and the head was placed in three-pin fixation with the head slightly extended. A left frontotemporal curvilinear incision was made behind the hairline. The temporalis fascia and muscle were preserved. A vascularized pericranial flap 


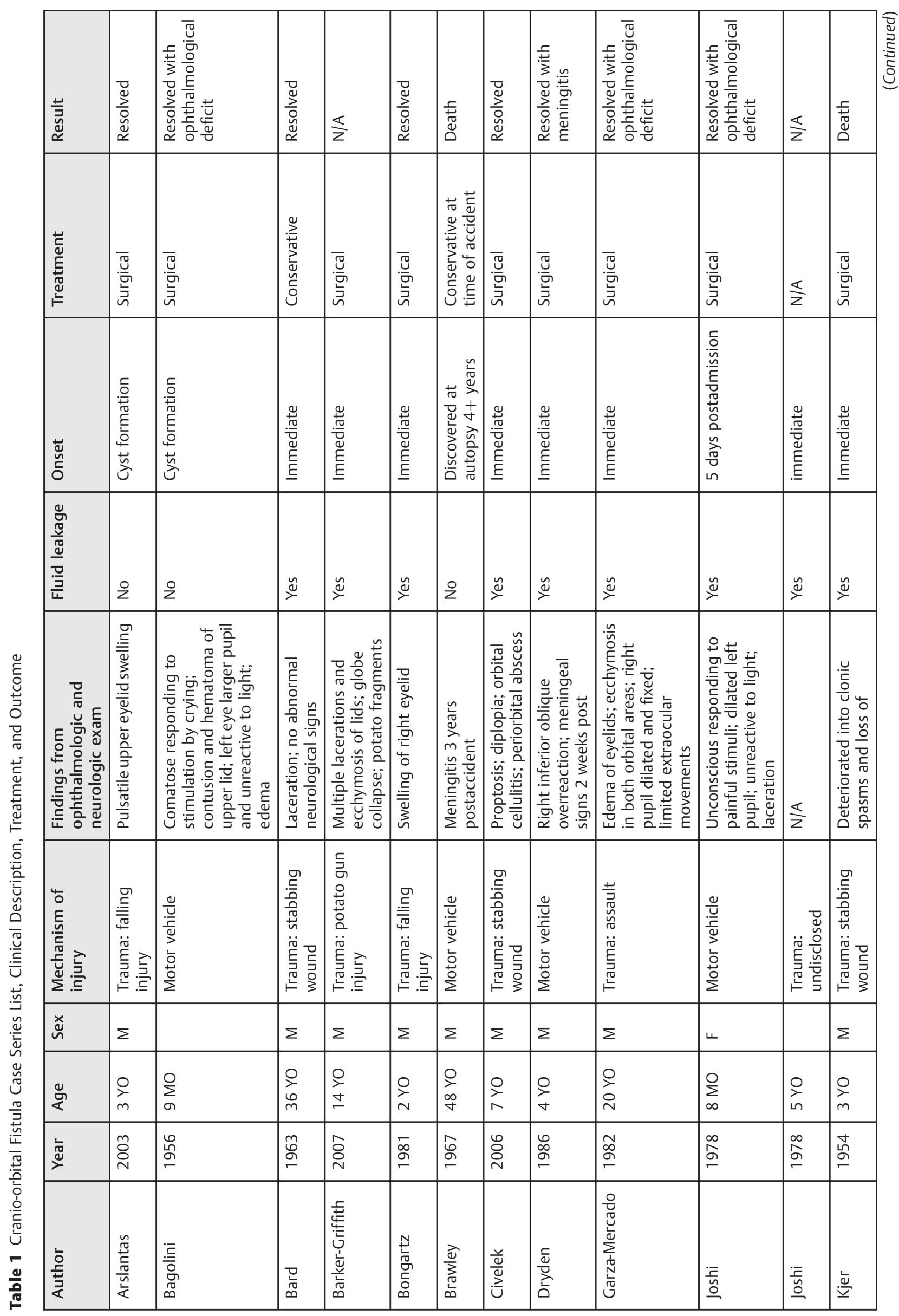




\begin{tabular}{|c|c|c|c|c|c|c|c|c|c|c|c|}
\hline 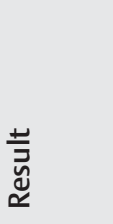 & & 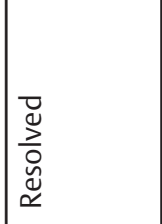 & 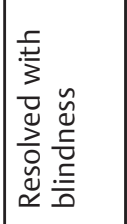 & 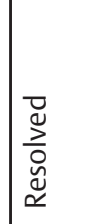 & 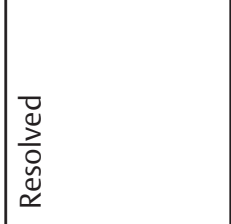 & $\begin{array}{l}\bar{D} \\
\stackrel{\Delta}{0} \\
\mathscr{y} \\
\check{u}\end{array}$ & 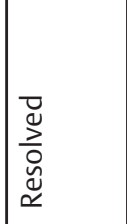 & $\begin{array}{l}\bar{D} \\
\stackrel{\Delta}{0} \\
\mathscr{y} \\
\check{\mathscr{u}}\end{array}$ & 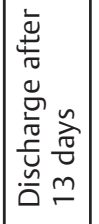 & 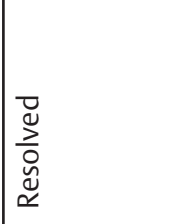 & $\mid \begin{array}{l}\vec{g} \\
\stackrel{\Xi}{0} \\
\check{y} \\
\check{y}\end{array}$ \\
\hline 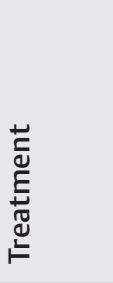 & & 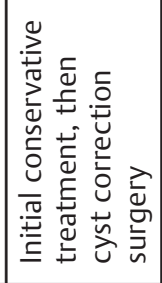 & | & 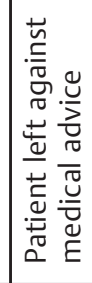 & 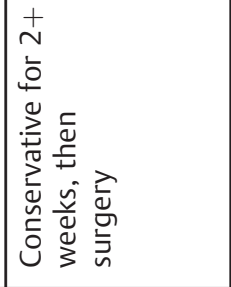 & 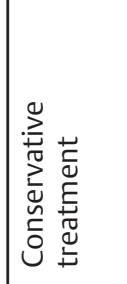 & 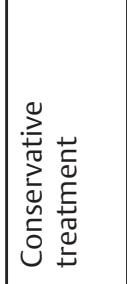 & 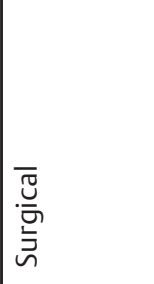 & $\begin{array}{l}\overline{\bar{v}} \\
\overline{\bar{n}} \\
\bar{\Xi}\end{array}$ & 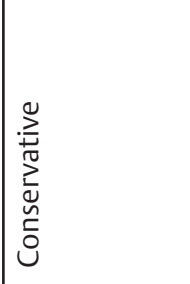 & $\begin{array}{l}\overline{\bar{U}} \\
\overline{\bar{n}} \\
\tilde{\Xi}\end{array}$ \\
\hline $\begin{array}{l}\text { ّ̆ } \\
\tilde{\Xi}\end{array}$ & & 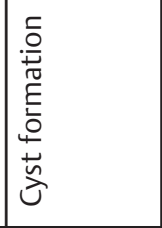 & 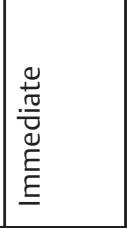 & 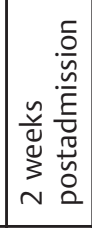 & 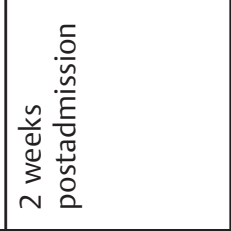 & 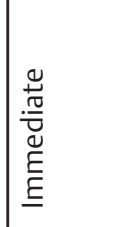 & 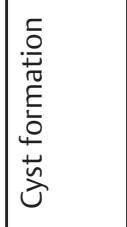 & 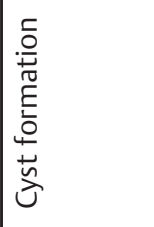 & & 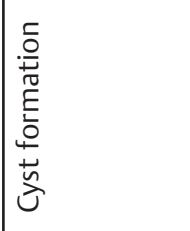 & 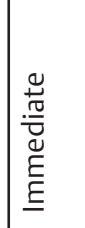 \\
\hline 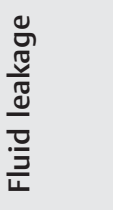 & & 2 & $\stackrel{\check{y}}{\rightleftharpoons}$ & $\stackrel{\check{\nu}}{\check{\nu}}$ & $\stackrel{\check{\nu}}{\rightleftharpoons}$ & $\stackrel{\check{\varphi}}{\rightleftharpoons}$ & 2 & i & & 2 & $\stackrel{\check{\varphi}}{\rightleftharpoons}$ \\
\hline 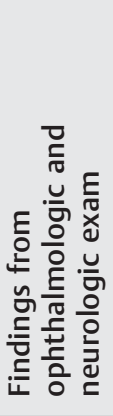 & 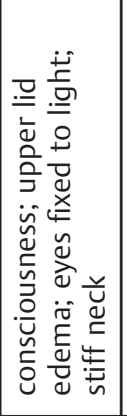 & 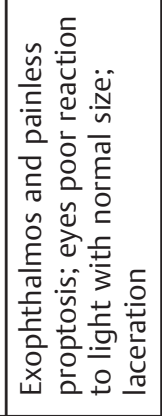 & 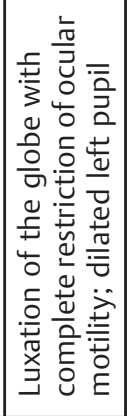 & 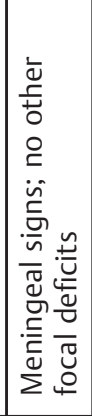 & 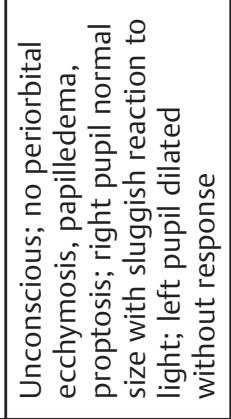 & 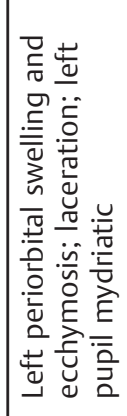 & 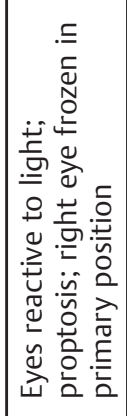 & 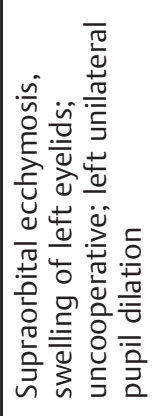 & 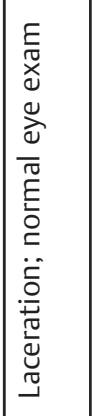 & 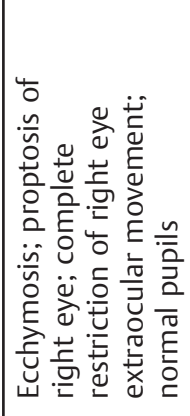 & 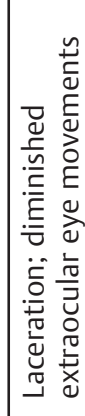 \\
\hline 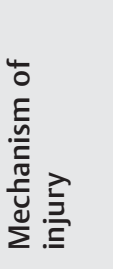 & & 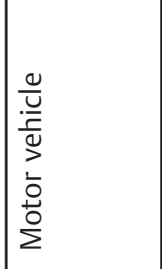 & 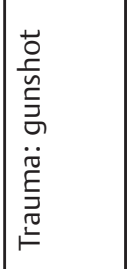 & 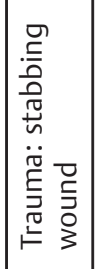 & 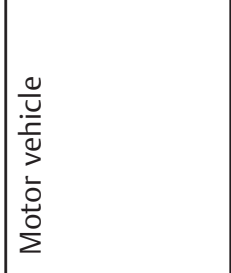 & 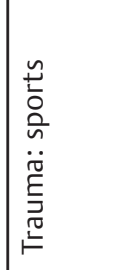 & 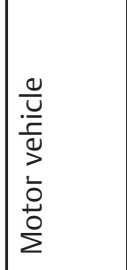 & 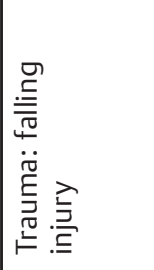 & 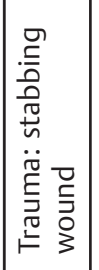 & 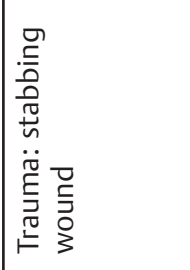 & 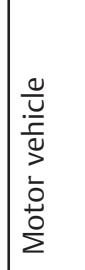 \\
\hline$\stackrel{\times}{\sim}$ & & ப & $\sqcup$ & $\Sigma$ & $\Sigma$ & $\sqcup$ & $\Sigma$ & $\Sigma$ & $\Sigma$ & $\Sigma$ & $\Sigma$ \\
\hline 苂 & & $\begin{array}{l}\stackrel{\ominus}{\succsim} \\
\bar{\gamma}\end{array}$ & $\sum_{\Gamma}^{O}$ & 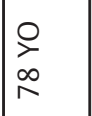 & 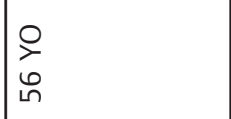 & 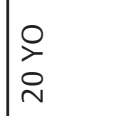 & $\begin{array}{l}\stackrel{\ominus}{\succ} \\
\searrow \\
\searrow\end{array}$ & $\sum_{\substack{0\\
}}$ & $\begin{array}{l}O \\
\sum \\
\pm\end{array}$ & $\stackrel{\rho}{\stackrel{\rho}{m}}$ & 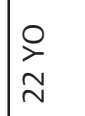 \\
\hline 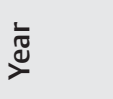 & & ஜ̊ & 总 & g & 站 & $\stackrel{\circ}{\stackrel{一}{~}}$ & $\begin{array}{l}\stackrel{\operatorname{l}}{\infty} \\
\stackrel{2}{\sigma}\end{array}$ & 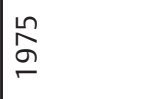 & $\begin{array}{l}\infty \\
\infty \\
\wp\end{array}$ & 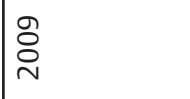 & $\frac{\sim}{\stackrel{N}{N}}$ \\
\hline 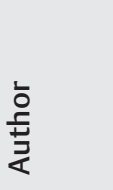 & & 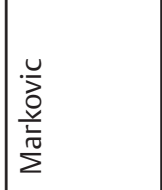 & 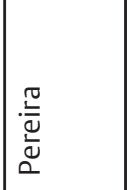 & 苂 & $\frac{\frac{\pi}{\alpha}}{\alpha}$ & 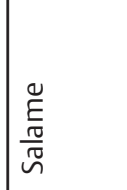 & $\begin{array}{l}\overrightarrow{\hat{\Xi}} \\
\text { ì } \\
\text { in }\end{array}$ & $\begin{array}{l}0 \\
\frac{\pi}{\pi} \\
\stackrel{0}{\omega}\end{array}$ & $\bar{\equiv}$ & $:=\bar{\pi}$ & $\mid \begin{array}{l}\ddot{\Delta} \\
\tilde{\Xi} \\
\tilde{\Sigma}\end{array}$ \\
\hline
\end{tabular}




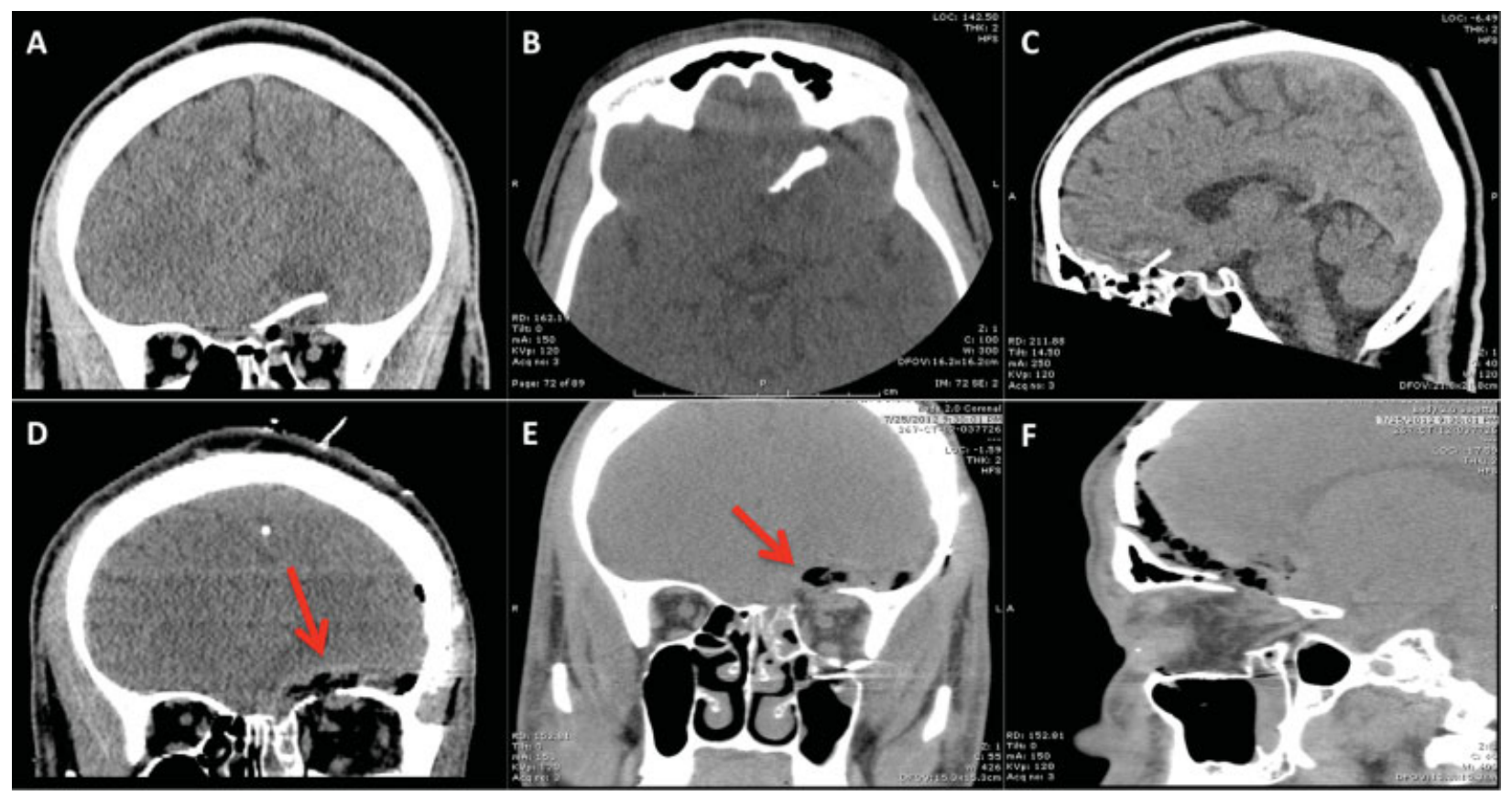

Fig. 1 Thin-cut preoperative and postoperative computerized tomography scans of the orbits with reconstructions. (A) Coronal view displaying superior orbit blowout fracture. (B) Axial view displaying bone fragment. (C) Sagittal view displaying superior displacement of bone fragment. (D) Coronal view showing interval resection of the displaced bone fragment. The red arrow denotes the pedicled pericranial flap. (E) Another coronal view showing both the integrity of the sinuses and the result of the surgical repair. The red arrow delineates the pericranial flap. (F) Sagittal view demonstrating the results of the surgical repair.

was harvested and preserved for subsequent reconstruction. A small $3 \times 2 \mathrm{~cm}$ craniotomy was made in the supraorbital region following single bur hole placement in the left anatomical keyhole. The posterior orbital rim was flattened using a highspeed drill. No frontal sinus breach was noted. The complex fracture was approached extradurally using gentle upward retraction of the left frontal lobe. A comminuted fracture of the left orbital roof was identified with severe herniation of the intraorbital contents (periorbital fat and muscle) into the frontal fossa. The sharp bony fragment displaced from the orbital roof had penetrated the dura and created a clear CSF fistula at this site. The bony fragment was gently resected from within the gyrus rectus parenchyma, and hemostasis was achieved using bipolar coagulation and oxidized cellulose. Following this, additional resection of the orbital roof was performed to further decompress the orbital contents. The large dural defect was repaired using a multilayer approach consisting of a synthetic dural graft inlay and overlay, in addition to application of a dural sealant (DuraSeal, Covidien, Mansfield, Massachusetts, USA). The vascularized pericranial flap was then placed as an overlay. The bone flap was replaced using standard low-profile titanium microplates. The wound was closed in standard multilayer fashion. Following this, the orbital floor fracture was repaired via a transconjunctival approach and reduction/fixation with titanium plates.

\section{Postoperative Course}

Fluid leakage ceased after completion of the surgery and repair of the dural compromise. The patient had a transient left third nerve palsy, which gradually improved over the next few weeks. At most recent follow-up 5 months later, the third nerve palsy had improved nearly completely with the exception of mild left ptosis, and the extraocular movements returned to normal with retention of full visual acuity in both eyes.

\section{Results}

A systematic review identified 22 cases of traumatic oculorrhea, which were included in the current analysis ${ }^{1-20}$ (-Table 1). The patient population had a mean age of 18.2 years with a median age of 7.5 years. Oculorrhea development was caused by a motor vehicle accident in eight patients, stab wounds through the orbit in six patients, falls in three patients, gunshot wounds in two patients, and one each of sports injury and undisclosed. Overall, the mechanism for oculorrhea formation was blunt head injury in 14 patients (64\%) and penetrating injury in 8 patients (36\%). Following ophthalmologic and neurologic exam, authors described the following relevant symptoms/signs: visible trauma to the face or orbit including lacerations, contusions, edema, ecchymosis (16), dilated pupil (7), unreactive to light (6), diminished ocular movements (6), altered consciousness (5), proptosis (5), meningitis signs (4), and diplopia (1). Seven patients underwent initial conservative treatment, with three patients ultimately needing surgical correction. Thirteen patients underwent initial surgical intervention. One patient left against medical advice, and one patient's outcome was not reported. 


\section{Discussion}

\section{Anatomical Considerations and Mechanisms of Injury}

The diagnosis of CSF oculorrhea may be challenging and go undetected despite careful neurological, orbital, and roentgenographic examinations. ${ }^{15}$ CSF leakage is often obfuscated by bleeding or severe ocular injury that commands the attention of the physician.

Some cases of oculorrhea are easily diagnosed. Of the 22 relevant cases, 8 were caused by penetrating wounds with obvious etiologies: 6 stab wounds and 2 gunshot wounds. For these cases, recognizing a penetrating injury as the mechanism is pivotal, and the diagnosis is typically made more easily. The foreign body passes through the orbit and into the cranium to directly penetrate the meninges and create a CSF fistula. ${ }^{6,9,19,20}$ Transorbital stab wounds are often fatal if no specific intervention is made. ${ }^{13}$ Characteristically, stab wounds have small external lesions with a long tract. This tract is often irregular from transient displacement of the tissue at the time of injury, creating an ideal location to grow anaerobic bacteria like Clostridium tetani. Appropriate antibiotics should be given immediately. Depending on the type and size of the penetrating wound, surgical intervention may be necessary to repair damage to the orbit and/or cranium.

In cases of blunt traumatic injury, unfamiliarity and complicated mechanisms frequently leave oculorrhea off of the physician's differential diagnosis. Several clues in the history may raise the suspicion for oculorrhea. Patients with cranioorbital fistulas from blunt traumatic injury tended to be involved in high-impact injuries, be young (median age 14 years), have an impaired ophthalmologic exam, and have variable onset of CSF oculorrhea.

The mechanism of injury provides some insight into the likelihood for a cranio-orbital fistula. Overall, most ocular injuries occur in home environments or car accidents. ${ }^{14}$ In this series, nine (56\%) occurred via a motor vehicle accident, six due to stab wounds (27\%), three (19\%) via a young-child falling accident, two via a gunshot wound (13\%), and one each of assault and sports trauma. Not surprisingly, these percentages closely follow reported mechanisms for injury in case series for CSF rhinorrhea. ${ }^{1}$ As expected, motor vehicle accidents and other high-impact mechanisms make up a majority of the overall cases for cranio-orbital fistulas.

The anatomy of the cranium and orbit provides some insight into the cause of cranio-orbital fistulas. The brain is enswathed from the exterior world by an anatomical barrier of skin, sinus mucosa, periosteum, skull bones, dura mater, and arachnoid membrane. Fistulas may develop when significant orbitocranial injuries breach the integrity of these barriers to permit the escape of CSF fluid. For true oculorrhea to occur with CSF exiting the orbit anteriorly, the continuity of both the meningeal and conjunctival layers must be compromised. ${ }^{15}$ This most frequently occurs in four anatomical locations along the skull base that may become structurally compromised: the frontal sinus, the cribriform plate/ethmoid roof, the sphenoid sinus, and the petrous bone. ${ }^{21}$

Four primary mechanisms provide an avenue for cranioorbital CSF communication. All, however, have anatomical considerations that seem to protect against the development of oculorrhea.

First, an orbital roof fracture provides a direct channel to the intracranial cavity. Bone fragments may frequently tear the dura and arachnoid but often leave the conjunctiva intact. In a majority of cases, CSF communicates between the two compartments but does not leak out of the eye. ${ }^{3}$ After severe breakage of this anatomical barrier, arachnoid membrane or frank brain tissue can herniate into the orbit, or orbital contents can herniate into the frontal fossa. In addition, during various orbital operations in which bone is removed from the skull base (such as invasive orbital tumor resection), development of CSF fistulas may occur. ${ }^{3}$

Second, concurrent damage to the cribriform plate and the medial wall of the orbit allows CSF to gain access to the orbit via the ethmoid air cells. The cribriform plate is a comparatively weak anatomical location susceptible to traumatic injury. The bone is thin and the dura invests from the olfactory fissure where the olfactory nerve penetrates the skull. Bone fragments from the ethmoid roof or orbital wall can easily tear the dura when fractured. ${ }^{10}$ CSF leaking through the cribriform plate, however, generally escapes into the paranasal sinuses and cavity to manifest as rhinorrhea rather than oculorrhea. This primarily derives from anatomical considerations: the bony walls of the air sinuses are thinner and break more easily than the orbital walls. ${ }^{3}$ The close proximity between the roof of the ethmoid and the nasal ostium, with only $5 \mathrm{~mm}$ separating the two, further encourages rhinorrhea as the predominant pathway of CSF leakage. Physiologically, the pressure gradient between the intracranial space and air sinuses is significantly greater than the gradient between the intracranial and intraorbital pressure. This further promotes development of rhinorrhea rather than oculorrhea as the "path of least resistance." Furthermore, patient age seems to be associated with the development of a cranio-orbital fistula. Overall, male children are the most likely group to have eye injuries. ${ }^{14}$ Most of the agespecific injuries, however, occur as a result of accidental trauma rather than the motor vehicle accidents prevalent in this series. Although younger children are at a high comparable risk for ocular injury, CSF rhinorrhea leaks are rare in children below the age of 2 , potentially resulting from the flexibility of the cranial base and the relative immaturity of the paranasal sinuses. ${ }^{10}$ Indeed, patients with larger sinuses are more at risk of developing rhinorrhea. ${ }^{4}$ In the current case series, three of the four youngest patients who developed oculorrhea in the non-stab wound category had an injury related to a fall, with an average age just under 2 years. Underdeveloped sinuses may anatomically promote cranioorbital fistulas compared with rhinorrhea by altering the route of CSF leak after damage to the cribriform plate. Galzio et al reported the case of a patient with frontal sinus agenesis who experienced cranio-orbital CSF leakage after a fracture of the anterior roof caused by blunt trauma. ${ }^{22}$ They hypothesized that the absence of a frontal sinus allowed the direct egress of CSF into the upper lid. A similar phenomenon may occur with underdeveloped sinuses in young children. Alternatively, the cribriform plate fracture is more likely to extend 
to the underdeveloped sinuses, as one case noted that the fracture line of the cribriform plate extended into the ethmoid air sinuses. ${ }^{12}$ The trend in this data may be an artifact of a small sample size or may indicate that underdeveloped sinuses alter the route of CSF leakage after traumatic injury. This may help to explain why three pediatric patients presented with this rare condition and why the median age of patients in our series reviews was so young.

Third, a CSF leak can remain trapped within the orbit as either an orbitocele or within the soft tissue of the eyelid itself. $^{2,4,5,18}$ This is a very rare phenomenon, with only five cases reported in the English literature. This can occur via accumulation of fluid in the orbit without conjunctival damage or through damage to another part of the calvarium with CSF seeping under the skin through a path into the eyelid. Cranio-orbital fistulas should be considered in patients presenting with orbitoceles or eyelid cysts who have a history of frontobasilar or superior orbital roof fractures. This is a complex diagnosis with many differential considerations, including a retrobulbar hematoma, orbital abscess, mucocele, or foreign body cyst. ${ }^{3}$ Lastly, CSF can leak via the optic canal when the arachnoid is damaged along with an orbital roof or apex injury. This is an uncommon mechanism and was not reported in this review.

\section{Clinical Presentation}

Symptoms and signs vary greatly in patients developing cranio-orbital fistulas. All but two patients had impaired ophthalmologic exams. The most common presenting symptoms/signs were visible trauma to the face or orbit including lacerations, contusions, edema, ecchymosis (81\%); epiphora or serous drainage from the eye (73\%); impaired papillary reflex and/or pupil dilation (47\%); orbital cyst formation (24\%); meningeal signs (19\%); loss of consciousness (19\%); loss of extraocular movements; and proptosis (19\%) (-Table 2). No distinct pattern of clinical findings, however, can unequivocally exclude or include a CSF cranio-orbital fistula. ${ }^{17}$ Meningeal signs may point to some type of fistula and may present without any focal neurological deficits. ${ }^{10} \mathrm{~A}$ history of prior surgery, especially if a portion of the orbital roof was removed, places patients at a higher risk for devel-

Table 2 List of Clinical Symptoms and Signs in Cranio-orbital Fistula Cases

\begin{tabular}{|l|l|}
\hline Symptom & Percent Presenting \\
\hline Loss of ocular movement & $19 \%$ \\
\hline Proptosis & $19 \%$ \\
\hline Orbital cyst formation & $24 \%$ \\
\hline Epiphora & $73 \%$ \\
\hline Visible damage & $81 \%$ \\
\hline Meningeal signs & $19 \%$ \\
\hline Loss of consciousness & $19 \%$ \\
\hline $\begin{array}{l}\text { Impaired pupillary reflex } \\
\text { or pupillary dilation }\end{array}$ & $47 \%$ \\
\hline
\end{tabular}

opment of cranio-orbital fistulas from subsequent trauma or surgeries. $^{23}$

A major cause of concern for cranio-orbital fistulas is external fluid leakage, as this increases the risk for meningitis or intracranial hypotension. The time course of CSF leakage can be quite variable. Due to the sparse oculorrhea data, clinical data of rhinorrhea onset is somewhat illustrative. Lewin et al showed that when CSF leakage occurred after head trauma, two thirds of the cases occurred within the first 48 hours. ${ }^{24}$ Most cases start within 3 months, but onset delays can range up to 30 years. In this review, 12 of the 15 cases presenting with oculorrhea had fluid leakage within 48 hours (80\%); one case presented at 5 days postadmission, and the other two cases presented at 2 weeks postadmission. ${ }^{8,12,16}$ Additional delayed imaging after fractures of the anterior skull base extending to the orbital roof or orbital walls may help identify delayed presentation of oculorrhea. This will help determine if a cranio-orbital fistula has developed after initial trauma.

Even in cases with immediate oculorrhea, identifying the discharged fluid as CSF may prove to be a challenge. In one case, the authors initially diagnosed posttraumatic fluid leak as epiphora due to lacrimal duct outflow. ${ }^{10}$ The 4 -year-old boy had a compromised lacrimal examination with drainage obstruction. His tearing failed to resolve after corrective lacrimal surgery, suggesting a cranio-orbital fistula as a possible underlying etiology or consequence of the lacrimal surgery. CSF fistulas should be a concern for all patients with excess tearing after anterior skull fractures or motor vehicle accidents.

Several means exist to differentiate epiphora from oculorrhea. First, analysis of the fluid for glucose content is illustrative. CSF normally has approximately two thirds of the serum glucose concentration, whereas tear glucose levels are insignificant (2.5 to $4.1 \mathrm{mg} / \mathrm{dL}){ }^{19}$ Glucose levels of $30 \mathrm{mg} / \mathrm{dL}$ or higher are diagnostic for oculorrhea. ${ }^{3}$ Each eye should be tested and compared, with any differences in glucose concentration noted. To date, no case of bilateral oculorrhea has been reported. Also, increased flow in the forward-tilting or otherwise head-dependent position may indicate a cranioorbital fistula resulting from positional pressure differences. Some authors discredit the use of glucose testing, claiming it is not as specific as immunoassays for $\beta 2$-transferrin. The latter test is both more specific and requires a smaller amount of fluid $(<1 \mathrm{~mL})$. Tears and nasal secretions lack protein, whereas CSF has significant levels. Together, these four indicators should aid to discriminate whether epiphora results from excess tear formation or a CSF leak.

The next step toward proper diagnosis is the selection of an appropriate imaging modality. CT imaging is the preferred method to screen for anterior skull base fractures with intracranial penetration. ${ }^{15}$ Thin-cut CT imaging with coronal and sagittal reconstructions provides an optimized view of the integrity of the skull base and is most useful for diagnosing orbital roof or other skull base fractures. In addition, CT scans are capable of detecting less than $0.5 \mathrm{~mL}$ of air within the cranial cavity (pneumocephalus), which is highly suggestive of a CSF fistula. Although 
localization of the CSF fistula may be a challenge, every effort to do so should be done prior to an operation. This can also be accomplished through metrizamide or iopamidol cisternography followed by thin-section coronal-view CT scanning. ${ }^{3}$ Radionucleotide cisternography is also reliable, yet not as sensitive of a method.

\section{Treatment of Cranio-Orbital Fistulas}

The optimal management of CSF orbital fistulas remains controversial based on the sparse frequency of this entity. The goal of fistula treatment is ideally to seal the hole in the dura/arachnoid, but this may not be necessary as long as there is no communication with the outside world or a sinus space. In a case of an orbital roof and superior orbital rim fracture without involvement of the paranasal sinuses, some CSF leakage may be reasonably expected into the orbit. ${ }^{18}$ For minor cases of CSF leakage, conservative treatment can often be attempted with success. Spontaneous healing and resolution of the CSF leak is more likely to occur if the dural edges remain in apposition without a major rent or if sinus mucosa bridges the dural defect. This allows for a plug to form in the dural hole. ${ }^{10}$ Conservative fistula management results in closure rates of $85 \%$ with bed rest alone, and up to 95 to $100 \%$ with spinal drainage in larger case series of rhinorrhea. ${ }^{3}$ In many cases of CSF oculorrhea, an attempt at conservative management is warranted. Twenty-five percent (5) of the patients in the current review were successfully treated with conservative management. Three patients resolved without complications, one patient experienced personality changes, and the last patient had meningitis 3 years postaccident. The small population size and large differences in diagnostic tools available allow little inference from this conservative treatment series.

In the current series, $14 \%$ of the patients (3) with initial conservative management failed and required subsequent surgery. Although some clinicians propose surgical intervention for all cases of external CSF leak to prevent later-onset infections of the central nervous system, the severity of the case typically determines the most appropriate treatment modality. ${ }^{3}$ Bony spicules protruding through a dural tear or brain herniation almost exclusively fail to heal conservatively and warrant surgical intervention. Additionally, coughing and straining may result in delayed CSF leakage or exacerbate a current CSF leakage, especially if the dura is injured and the arachnoid is at risk for delayed injury. ${ }^{10}$ Perhaps due to publication bias, most cases in the published English literature were severe cases that frequently necessitated surgical intervention as the primary intervention (60\%; 12 patients). All of these cases resolved the CSF leak, with complications including ophthalmological deficits (4), blindness (1), and meningitis (1).

Surgical intervention can involve either extracranial or intracranial approaches. The intracranial intradural approach is preferred for traumatic CSF fistulas to enable careful patching of the defect with autologous, pericranium, and/or fascia lata. ${ }^{15}$ During the surgery, accurate localization of the CSF fistula can be challenging task. ${ }^{25}$ The use of intra- operative intrathecal fluorescein (IF) may facilitate the localization of skull base defects and CSF leakage, as well as confirming a watertight closure of the leak after completion of the repair. In the patient presented in the current study, a large bone fragment derived from the fractured orbital roof was piercing the dura and gyrus rectus of the frontal lobe, which posed a major risk for subsequent seizures, a nonhealing fistula, and meningitis. The refractory nature of his CSF oculorrhea after 5 days of conservative management, in combination with the fracture pattern, made surgical intervention warranted. Based on this review, conservative management is appropriate for less severe cases of oculorrhea, with surgical management as the preferred method for all severe or refractory cases characterized by the predescribed criteria above.

\section{Conclusion}

Patients with craniofacial injury and fractures involving the anterior skull base and orbit may develop oculorrhea. The case presented in this paper provides an exemplar for diagnosing and surgically repairing refractory cranio-orbital fistulas with oculorrhea. Physicians should remain aware of cranio-orbital fistulas as a possible complication of severe anterior skull based fractures or iatrogenic surgical injury. Although attempted conservative management is warranted in a majority of cases, severe cases with displaced bone penetrating the dura and/or parenchyma or refractory oculorrhea should be treated surgically. Prompt diagnosis and correction should minimize the occurrence of complications including meningitis postinjury.

\section{References}

1 Bongartz EB, Nau HE, Liesegang J. The cerebrospinal fluid fistula. Rhinorrhoea, otorrhoea and orbitorrhoea. Neurosurg Rev 1981; 4(4):195-200

2 Arslantaş A, Vural M, Atasoy MA, Ozsandik A, Topbaş S, Tel E. Posttraumatic cerebrospinal fluid accumulation within the eyelid: a case report and review of the literature. Childs Nerv Syst 2003; 19(1):54-56

3 Salame K, Segev Y, Fliss DM, Ouaknine GE. Diagnosis and management of posttraumatic oculorrhea. Neurosurg Focus 2000;9(1):e3

4 Marković MV, Brkljacić B, Ratković NA, Vilendecić M, Franćeski D, Curić J. Posttraumatic cerebrospinal fluid cyst of the orbit. J Craniofac Surg 2006;17(1):189-191

5 Bagolini B. Leakage of spinal fluid into upper lid following trauma. AMA Arch Opthalmol 1957;57(3):454-456

6 Bard LA, Jarrett WH. Intracranial complications of penetrating orbital injuries. Arch Ophthalmol 1964;71:332-343

7 Barker-Griffith AE, Streeten BW, Abraham JL, Schaefer DP, Norton SW. Potato gun ocular injury. Ophthalmology 1998;105(3): 535-538

8 Brawley BW, Kelly WA. Treatment of basal skull fractures with and without cerebrospinal fluid fistulae. J Neurosurg 1967;26(1): 57-61

9 Civelek E, Bilgiç S, Kabataş S, Hepgül KT. [Penetrating transorbital intracranial foreign body]. Ulus Travma Acil Cerrahi Derg 2006; 12(3):245-248

10 Dryden RM, Wulc AE. Pseudoepiphora from cerebrospinal fluid leak: case report. Br J Ophthalmol 1986;70(8):570-574 
11 Garza-Mercado R, Aragon-Lomas J, Martinez-Garza J, LealHernandez L. Cerebrospinal fluid blepharocele: an unusual complication of head injuries. Neurosurgery 1982;11(4): 525-526

12 Joshi KK, Crockard HA. Traumatic cerebrospinal fluid fistula simulating tears. Case report. J Neurosurg 1978;49(1):121-123

13 Kjer P. Orbital and transorbital stab wounds. AMA Arch Opthalmol 1954;51(6):811-821

14 Pereira FJ, Bettega RB, Velasco e Cruz AA. Management of globe luxation followed by traumatic liquoric fistula: case report. Arq Bras Oftalmol 2011;74(1):58-60

15 Rao TN, Purohit AK, Dilnawaz, Murthy TV, Dinakar I. CSF orbitorrhoea with tension pneumocephalus. Neurol India 1999;47(1): 65-67

16 Rha EY, Kim JH, Byeon JH. Posttraumatic delayed cranio-orbital cerebrospinal fluid leakage: Case report. J Plast Reconstr Aesthet Surg 2013;66(4):563-565

17 Sibony PA, Anand AK, Keuskamp PA, Zippen AG. Posttraumatic cerebrospinal fluid cyst of the orbit. Case report. J Neurosurg 1985;62(6):922-924
18 Terao H, Sato S. Ventriculo-orbital fistula in closed head injury. Case report. J Neurosurg 1975;43(6):754-756

19 Till JS, Marion JR. Cerebrospinal fluid masquerading as tears. South Med J 1987;80(5):639-640

20 Twaij S, Viswanathan P, Page AB. Acute traumatic orbital cerebrospinal fluid cystocele mimicking orbital abscess. J AAPOS 2009; 13(5):491-493

21 McCormack B, Cooper PR, Persky M, Rothstein S. Extracranial repair of cerebrospinal fluid fistulas: technique and results in 37 patients. Neurosurgery 1990;27(3):412-417

22 Galzio RJ, Lucantoni D, Zenobii M, Grizzi LC. Traumatic craniopalpebral cerebrospinal fluid fistula. J Neurosurg Sci 1981;25(2):105-107

23 King AB. Traumatic encephaloceles of the orbit. AMA Arch Opthalmol 1951;46(1):49-56

24 Lewin W. Cerebrospinal fluid rhinorrhea in nonmissile head injuries. Clin Neurosurg 1964;12:237-252

25 Seth R, Rajasekaran K, Benninger MS, Batra PS. The utility of intrathecal fluorescein in cerebrospinal fluid leak repair. Otolaryngol Head Neck Surg 2010;143(5):626-632 